\title{
Application of personal-oriented digital technology in preventing transmission of COVID-19, China
}

\author{
Xiao-Ben $\operatorname{Pan}^{1}$ \\ Received: 9 March 2020 / Accepted: 13 March 2020 / Published online: 27 March 2020 \\ (C) Royal Academy of Medicine in Ireland 2020
}

\begin{abstract}
We reported several personal-oriented and mobile phone-based information technologies which were recently developed and widely used during the outbreak of COVID-19 in China. These technologies help reduce the transmission of COVID-19 and maintain normal social order.
\end{abstract}

Keywords Coronavirus $\cdot$ Information technology $\cdot$ Prevention

\section{To Editor}

Information technology has been widely used by medical professionals in the hospitals and the centers of disease control for the prevention and control of infectious disease, which includes timely reporting, epidemic surveillance, and monitoring of programmed immunization. In addition, web-based surveillance tools and epidemic intelligence methods, used by public health institutions, are intended to facilitate risk assessment and timely outbreak detection. However, information technology is poorly developed for use by general people, although they are actually the susceptible subjects of infection and the infectious source as well.

The outbreak of coronavirus disease 2019 (COVID19), a disease stemming from SARS-COV-2, began in the year 2019 December in Wuhan, China. Currently, it has since spread to over 100 thousand people in over 100 countries around the world. To prevent a massive outbreak of the disease in China, the Chinese government has adopted sweeping prevention measures, including lockdown and travel restriction in several cities. Although these measures significantly help prevent the transmission of disease, it also causes inconvenience to

Xiao-Ben Pan

panxiaoben@hznu.edu.cn

1 Department of Basic Medical Science, Key Laboratory of Aging and Cancer Biology of Zhejiang Province, Key Laboratory of Inflammation and Immunoregulation of Hangzhou, School of Medicine, Hangzhou Normal University, No.2318, Yuhangtang Rd, Cangqian, Yuhang District, 311121 Hangzhou, Zhejiang, People's Republic of China the lives of general people. During this period, several personal-oriented and mobile phone-based information technologies are developed and widely used in China, which helps reduce the transmission of COVID-19 and maintain normal social order.

The application of health status code first launched on 11th Feb in Hangzhou city, Zhejiang, China. Based on big data and mobile internet technologies, residents and those entering the city need to apply online and receive either a green, red, or yellow code. The colors are based on the information reported by the applicants, including their state of health, travel history, and whether they contacted people from epidemic areas. People with green code may travel around the city. Those with red or yellow codes must first undergo 7 to 14 days of quarantine. According to the classification, the government restricts the travel of people with potential infection but allow healthy people to travel freely and resume work. Based on a summary of local experience, the Chinese government promoted a unified health code system throughout the country.

Tremendous population migration during the period of Chinese New Year significantly promoted the nationwide spread of COVID-19. An application titled Query of Same Itinerary with Patient came online. The application timely updates the information about public transportation that carried the patients, including airline, railway, and city traffic. The passengers can actively search whether the transportation they boarded has a COVID-19 patient. In addition, the system will send messages to the passenger who has been close to the patients in transportation. With the authority by the passenger, the system can detect their train itineraries for the last 30 days. If there is a COVID-19 patient on the same itinerary, the passengers will receive notifications immediately. 
The application of Diagnosed Cases in Community enables people to check the distribution of COVID-19 cases in local communities on the map. The map covers more than 130 cities in China and shows case number and location. This App help people carefully manage their surrounding travel and review potential contact with the patients.

In conclusion, different from the traditional information technologies used by medical professionals, this kind of novel personal-oriented digital technology can be easily used by the public and help individuals assess and alert to the risk of transmission. In addition, it facilitates the government to conduct crisis management during the outbreak period. Although further improvement would be needed, we recommended that personal-oriented digital technology may help cut down the spread of COVID-19 in the areas with pandemic and maintain the local social order in current emergency. In addition, we suggested that the personal-oriented digital platform might be included as a component of prevention and control system against infectious diseases in the future.

Funding information This study was supported by grants from the National Natural Science Foundation of China (81670530), the Start-up Foundation (2018QDL035), and Pandeng Project of Hangzhou Normal University.

Publisher's note Springer Nature remains neutral with regard to jurisdictional claims in published maps and institutional affiliations. 